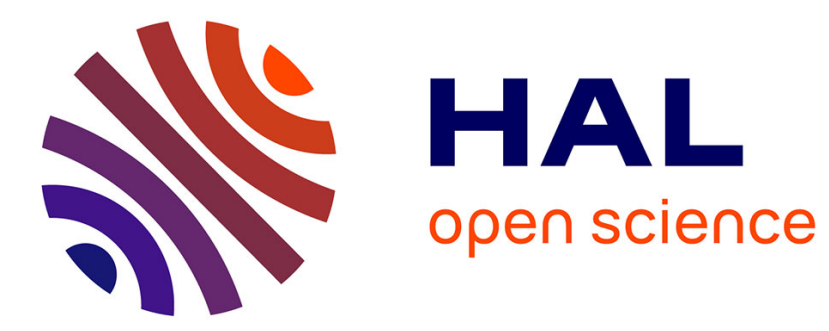

\title{
Dualization on Partially Ordered Sets: Preliminary Results
}

\author{
Lhouari Nourine, Jean-Marc Petit
}

\section{To cite this version:}

Lhouari Nourine, Jean-Marc Petit. Dualization on Partially Ordered Sets: Preliminary Results. Communications in Computer and Information Science, Springer, pp.23-34, 2015, International Workshop, ISIP 2014, Kuala Lumpur, Malaysia, October 11-13, 2014. Revised Selected Papers. hal-01229022

\section{HAL Id: hal-01229022 \\ https://hal.science/hal-01229022}

Submitted on 16 Nov 2015

HAL is a multi-disciplinary open access archive for the deposit and dissemination of scientific research documents, whether they are published or not. The documents may come from teaching and research institutions in France or abroad, or from public or private research centers.
L'archive ouverte pluridisciplinaire HAL, est destinée au dépôt et à la diffusion de documents scientifiques de niveau recherche, publiés ou non, émanant des établissements d'enseignement et de recherche français ou étrangers, des laboratoires publics ou privés. 


\title{
Dualization on partially ordered sets: Preliminary Results
}

\author{
Lhouari Nourine $^{1}$ and Jean Marc Petit ${ }^{2}$ \\ 1 Clermont-Université, Université Blaise Pascal, LIMOS, CNRS, France \\ 2 Université de Lyon, CNRS, LIRIS, INSA Lyon, France
}

\begin{abstract}
The dualization problem on arbitrary posets is a crucial step in many applications in logics, databases, artificial intelligence and pattern mining.

The objective of this paper is to study reductions of the dualization problem on arbitrary posets to the dualization problem on boolean lattices, for which output quasi-polynomial time algorithms exist. We introduce convex embedding and poset reflection as key notions to characterize such reductions. As a consequence, we identify posets, which are not boolean lattices, for which the dualization problem remains quasi-polynomial and propose a classification of posets with respect to dualization.

As far as we know, this is the first contribution to explicit non-trivial reductions for studying the hardness of dualization problems on arbitrary posets.
\end{abstract}

\section{Introduction}

The dualization problem in arbitrary finite ${ }^{3}$ partially ordered sets (poset for short), is a crucial step in many applications in logics, databases, artificial intelligence and pattern mining and has been intensively studied for years [1-3]. The dualization problem can be very difficult and the decision problem associated to dualization is still open, even for lattices. Only a few results exist, mainly dualization is quasi-polynomial whenever $(P, \leq)$ is isomorphic to a powerset ordered under set inclusion (or boolean lattice) [2], which corresponds to the enumeration of minimal transversal of hypergraph.

The dualization problem on hypergraphs has been studied by many researchers, among which we quote $[1,2]$ while only a few results exist on dualization on other posets [4]. Recently Kante et al [5] have shown that the enumeration of minimal transversal of an hypergraph is equivalent to the enumeration of minimal dominating sets of a cobipartite graph. Interestingly, this result brings the dualization problem to the large graph theory community.

Some theoretical frameworks for pattern mining have studied dualization, for instance [6-8]. In [8], we introduced the idea of weak representations as sets for pattern mining problems and showed how the frequent rigid sequences mining

\footnotetext{
${ }^{3}$ It also works for infinite partially ordered sets that are well ordered, i.e. all antichains
} are finite. 
problem benefits from such representation. The embryo of a reduction for the dualization problem on arbitrary posets was present but was clearly implicit in [8].

To measure the complexity of enumeration algorithms, we always refer to the complexity in the size of the input and the size of the output, see [9] for details.

The objective of this paper is to study reductions of the dualization problem on arbitrary posets to the dualization problem on boolean lattices. On posets, the dualization problem can be stated as follows:

\section{DualizeOnPoset}

Input: A representation of a poset $(P, \leq), B^{+}$an antichain of $P$.

Output: $B^{-}$such that $\left(B^{+}, B^{-}\right)$are dual $\operatorname{sets}^{4}$ in $P$.

Let us consider the particular instance of this problem:

\section{DualizeOnSet}

Input: A finite set $E, B^{+}$an antichain of $\mathcal{P}(E)$ (the powerset of $E$ ).

Output: $B^{-}$such that $\left(B^{+}, B^{-}\right)$are dual sets in $\mathcal{P}(E)$.

As already mentioned, the complexity of DualizeOnSet is known to be quasi-polynomial while the complexity of DualizeOnPoset is still open in most posets (for example, lattice) [4]. In this setting, we are interested in studying the reduction from DualizeOnPoset to DualizeOnSet, i.e. under which conditions DualizeOnSet is at least as hard as DualizeOnPoset. Notice that reductions for the hardness of enumeration problems are not well established as for decision problems. In this paper, we consider only polynomial time reduction as explained in Figure 1 which is inspired from classical polynomial reduction of decision problems.

Contribution on dualization : We introduce convex embedding and poset reflection as key notions to characterize such reductions. As a consequence, we identify posets, which are not boolean lattices, for which the dualization problem remains quasi-polynomial and propose a classification of posets with respect to dualization.

\section{Preliminaries}

We briefly recall definitions on partial orders, embeddings and borders $[10,8]$.

A partial order is a binary relation $\leq$ over a set $P$ which is reflexive, antisymmetric, and transitive. Let $x, y$ be elements of $P$, if $x \leq y$ or $y \leq x$, then $x$ and $y$ are comparable, otherwise they are incomparable. A partial order under which every pair of elements is comparable is called a chain. A subset of a poset in which no two distinct elements are comparable is called an antichain. We say that $y$ covers $x$ if whenever $x \leq z \leq y$ then $z=x$ or $y=z$; we denote by $\prec$ the covering relation. For $S \subseteq P, \downarrow S$ (resp. $\uparrow S$ ) is the downward (resp. upward) closed set of $S$ under the relation $\leq$ (i.e. $\downarrow S$ is an ideal and $\uparrow S$ a filter of $(P, \leq)$ ). In case of ambiguity, $\downarrow S$ (resp. $\uparrow S$ ) will be denoted by $\downarrow \leq S$ (resp. $\uparrow \leq S$ ). A

\footnotetext{
${ }^{4}$ Dual sets are also known as blocker and anti-blocker or positive and negative borders
} 


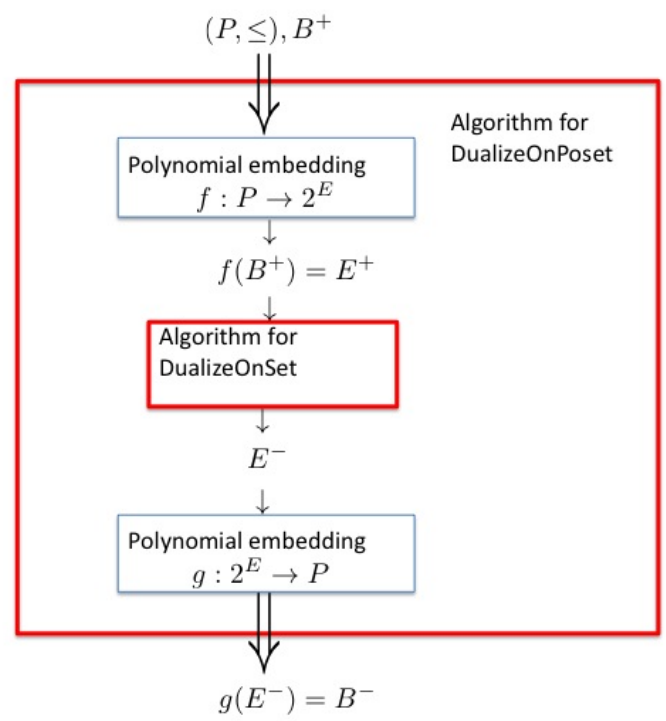

Fig. 1. Reduction from DualizeOnPoset to DualizeOnSet

subset $S \subseteq P$ is convex in $P$ if for all $x, y, z \in P, x, y \in S$ and $x \leq z \leq y$ implies $z \in S$. We denote by $\operatorname{Max}_{\leq}(S)$ (resp. $\operatorname{Min}_{\leq}(S)$ ) denotes the maximal (resp. minimal) elements of $S$ with respect to $\leq$. When $\leq$ is clear from context, $(P, \leq)$ $\left(\right.$ resp. $\operatorname{Max}_{\leq}(S)$ and $\left.\operatorname{Min}_{\leq}(S)\right)$ will be denoted by $P(\operatorname{resp} . \operatorname{Max}(S), \operatorname{Min}(S))$.

Let $\left(P, \leq_{P}\right)$ and $\left(Q, \leq_{Q}\right)$ be posets and $f: P \rightarrow Q$ a mapping (total function). $f$ is an embedding if for all $x, y \in P, x \leq_{P} y$ iff $f(x) \leq_{Q} f(y)$. The mapping $f$ is an isomorphism if $f$ is a bijective embedding. In this case $P$ and $Q$ are said to be isomorphic. $f$ is a convex embedding if $f$ is an embedding and $f(P)$ is convex in $\left(Q, \leq_{Q}\right)$. Whenever $f$ is injective but not surjective, there exists another mapping $g: f(P) \rightarrow P$ such that $g \circ f=I d$, the identity function. A reflection of a poset $(P, \leq)$ is a poset $\left(P, \leq^{\prime}\right)$ on the same ground set $P$ such that for all $x, y \in P, x \leq^{\prime} y \Rightarrow x \leq y$.

Two antichains $\left(\mathcal{B}^{+}, \mathcal{B}^{-}\right)$of $P$ are said to be dual if $\downarrow \mathcal{B}^{+} \cup \uparrow \mathcal{B}^{-}=P$ and $\downarrow \mathcal{B}^{+} \cap \uparrow \mathcal{B}^{-}=\emptyset$. The relationship between these dual sets is known as the dualization, i.e. given $\mathcal{B}^{+}$, compute $\mathcal{B}^{-}$(or inversely). In the sequel, $\left(\mathcal{B}^{+}, \mathcal{B}^{-}\right)$ will be referred to as a "border".

Let $f: P \rightarrow Q$ be a mapping and $\left(\mathcal{B}^{+}, \mathcal{B}^{-}\right)$a border in $P$. The border $\left(\mathcal{E}^{+}, \mathcal{E}^{-}\right)$in $Q$ is an extension of $\left(\mathcal{B}^{+}, \mathcal{B}^{-}\right)$with respect to $f$, if $f\left(\mathcal{B}^{+}\right) \subseteq \mathcal{E}^{+}$and $f\left(\mathcal{B}^{-}\right) \subseteq \mathcal{E}^{-}$. The extension $\left(\mathcal{E}^{+}, \mathcal{E}^{-}\right)$is said to be a polynomial extension of $\left(\mathcal{B}^{+}, \mathcal{B}^{-}\right)$if $\left|\mathcal{E}^{+}\right|+\left|\mathcal{E}^{-}\right|$is polynomial in $\left|\mathcal{B}^{+}\right|+\left|\mathcal{B}^{-}\right|$. 
The intuition of the reduction of enumeration problems used in this paper is based on finding a mapping between posets such that borders are polynomial preserved, i.e. every border has a polynomial extension.

In the rest of this paper, we assume that a poset is given by an implicit representation $\mathcal{L}$ and we shall denote by $\left(\mathcal{L}^{*}, \leq\right)$ the poset defined by $\mathcal{L}$. Clearly, the size of $\mathcal{L}^{*}$ may be exponential in the size of the representation $\mathcal{L}$. For instance, the free monoid $\Sigma^{*}$ ordered by sub word is implicitly defined by the alphabet $\Sigma$, and a lattice is implicitly defined by its poset of (join and meet) irreducible elements[11].

\section{Classification of posets with respect to dualization}

In this section we describe two properties of posets that lead us to have polynomial time reductions to DualizeOnSet. First we show that a convex embedding from a poset $\left(\mathcal{L}^{*}, \preceq\right)$ to $\mathcal{P}(E)$ for some set $E$ is sufficient to re-use algorithms of DualizeOnSet. Second, we show that the convex embedding is not a necessary condition and introduce the reflection of a poset $\left(\mathcal{L}^{*}, \preceq\right)$ to obtain a new poset $\left(\mathcal{L}^{*}, \preceq^{\prime}\right)$ in which there is a convex embedding. Indeed, a reflection of a poset $\left(\mathcal{L}^{*}, \preceq\right)$ corresponds to an embedding which preserves incomparabilities (or antichains), even if some comparabilities could be lost. The previous two embeddings introduce extra-elements to the dualization. Whenever these extra-elements are bounded by a polynomial, the dualization can be polynomial reduced to DualizeOnSet. To do so, we ask the following questions:

Given a poset of patterns $\left(\mathcal{L}^{*}, \preceq\right)$,

- Does there exist a convex embedding of $\left(\mathcal{L}^{*}, \preceq\right)$ into $(\mathcal{P}(E), \subseteq)$ for some finite set $E$ ? If not,

- Does there exist a reflection $\left(\mathcal{L}^{*}, \preceq^{\prime}\right)$ of $\left(\mathcal{L}^{*}, \preceq\right)$ such that there exists a convex embedding of $\left(\mathcal{L}^{*}, \preceq^{\prime}\right)$ into $(\mathcal{P}(E), \subseteq)$ for some finite set $E$ ?

These two questions and their associated computational costs allow to come up with new classes of posets with respect to dualization. Figure 2 gives an illustration with a diagram where posets and borders are placed side by side.

\subsection{Convex embedding}

First, let us recall that any poset has an embedding into a boolean lattice.

Proposition 1. [10] For any poset $\left(\mathcal{L}^{*}, \preceq\right)$, there exists an embedding from $\left(\mathcal{L}^{*}, \preceq\right)$ to $(\mathcal{P}(E), \subseteq)$, for some finite set $E$.

It follows that any poset has a set representation but obviously the dualization on $\left(\mathcal{L}^{*}, \preceq\right)$ may be much more complex than the dualization on $(\mathcal{P}(E), \subseteq)$ [8]. We define the $\mathcal{R} \mathcal{A S}$ class as follows:

Definition 1. $\left(\mathcal{L}^{*}, \preceq\right) \in \mathcal{R} \mathcal{A S}$ iff $\left(\mathcal{L}^{*}, \preceq\right)$ and $(\mathcal{P}(E), \subseteq)$ are isomorphic, for some finite set $E$. 


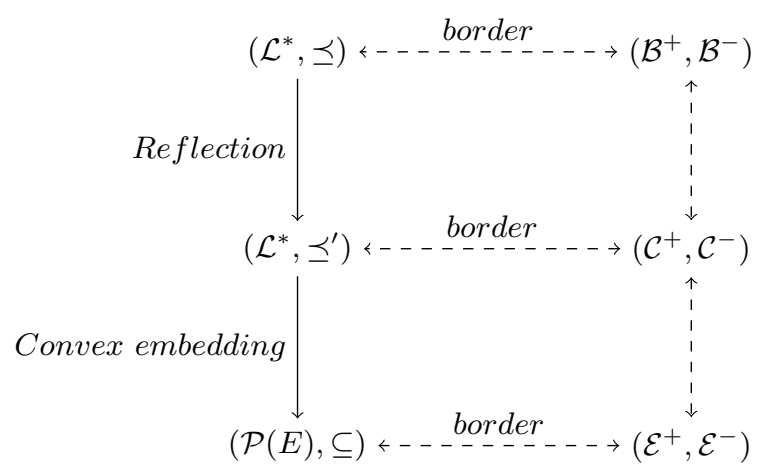

Fig. 2. Reflection and convex embedding

This class of posets gathers together many patterns such as frequent itemsets (FIM) [12], functional dependencies (FD) [13], inclusion dependencies (IND) [14]. This class is known as the representation as sets class of pattern mining problems defined in [6].

Nevertheless, requirements to be in $\mathcal{R} \mathcal{A S}$ are restrictive, since the poset must be isomorphic to a boolean lattice, and then its size has to be equal to $2^{n}$ where $n=|E|$. Now we will relax the bijective constraint of $\mathcal{R} \mathcal{A S}$ but we keep the convexity property on the set representation. Hence, we extend $\mathcal{R} \mathcal{A S}$ to a new class, called $\mathcal{X} \mathcal{R} \mathcal{A S}$, for conve $\mathcal{X} \mathcal{R} \mathcal{A S}$.

Definition 2. $\left(\mathcal{L}^{*}, \preceq\right) \in \mathcal{X} \mathcal{R} \mathcal{A S}$ iff there exists a convex embedding from $\left(\mathcal{L}^{*}, \preceq\right.$ ) to $(\mathcal{P}(E), \subseteq)$, for some finite set $E$.

The idea is still to require an isomorphism but just between the poset of patterns and some subset of $\mathcal{P}(E)$, instead of the entire set $\mathcal{P}(E)$ (see Figure 3). Note also that $f$ is injective since $f$ is an embedding. The following proposition points out a simple yet important characterization of $\mathcal{X} \mathcal{R} \mathcal{A S}$ problems.

Proposition 2. $\left(\mathcal{L}^{*}, \preceq\right) \in \mathcal{X} \mathcal{R} \mathcal{A S}$ iff $\left(\mathcal{L}^{*}, \preceq\right)$ is isomorphic to $\left(\mathcal{P}(E) \backslash\left(\downarrow B_{0}^{+} \cup \uparrow\right.\right.$ $\left.\left.B_{0}^{-}\right), \subseteq\right)$ for some antichains $B_{0}^{+} \subseteq \mathcal{P}(E)$ and $B_{0}^{-} \subseteq \mathcal{P}(E)$.

Proof. Let $f$ be a convex embedding from $\left(\mathcal{L}^{*}, \preceq\right)$ to $(\mathcal{P}(E), \subseteq)$ and $\mathcal{F}=f\left(\mathcal{L}^{*}\right)$. Let us consider $\mathcal{F}^{+}=\operatorname{Min}(\mathcal{F})$ and $\mathcal{F}^{-}=\operatorname{Max}(\mathcal{F})$ two antichains of $\mathcal{F}$. Moreover, let $B_{0}^{+}=\operatorname{Max}\left(\left\{X \in \mathcal{P}(E) \mid X \subset Y, Y \in F^{+}\right\}\right)$and $B_{0}^{-}=\operatorname{Min}(\mathcal{P}(E) \backslash$ $\left.\left(\mathcal{F} \cup \downarrow B_{0}^{+}\right)\right)$. Let $X \in \mathcal{P}(E)$. Then either $X \in \downarrow B_{0}^{+}$, or $X \in \uparrow B_{0}^{-}$or $X \in$ $\mathcal{P}(E) \backslash\left(\downarrow B_{0}^{+} \cup \uparrow B_{0}^{-}\right)$. In the latter case, there exists $Y_{1} \in F^{+}, Y_{2} \in F^{-}$such that $Y_{1} \subseteq X \subseteq Y_{2}$. Since $\mathcal{F}$ is convex, $X \in \mathcal{F}$ and the result follows.

The other direction holds since $\mathcal{P}(E) \backslash\left(\downarrow B_{0}^{+} \cup \uparrow B_{0}^{-}\right)$is a convex set of $\mathcal{P}(E)$.

Figure 3 gives an illustration of the proposition 2 .

Note that the sets $B_{0}^{+}$and $B_{0}^{-}$can be exponential in the size of $E$. The next definition introduces efficient problems of $\mathcal{X} \mathcal{R} \mathcal{A S}$, called $\mathcal{E} \mathcal{X} \mathcal{A S}$. 


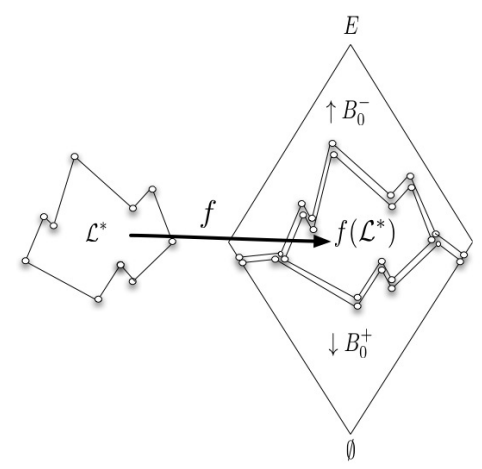

Fig. 3. The class $\mathcal{X} \mathcal{R} \mathcal{A S}$

Definition 3. $\left(\mathcal{L}^{*}, \preceq\right) \in \mathcal{E} \mathcal{X} \mathcal{R} \mathcal{A S}$ if $\left(\mathcal{L}^{*}, \preceq\right) \in \mathcal{X} \mathcal{R} \mathcal{A S}$ and $\left|B_{0}^{+} \cup B_{0}^{-}\right|$is polynomial in $|E|$.

The following proposition points out that a polynomial extension of any border of $\left(\mathcal{L}^{*}, \preceq\right)$ exists if $\left(\mathcal{L}^{*}, \preceq\right) \in \mathcal{E} \mathcal{X} \mathcal{R} \mathcal{A S}$.

Proposition 3. Let $\left(\mathcal{L}^{*}, \preceq\right) \in \mathcal{E} \mathcal{X} \mathcal{R} \mathcal{A S}$ and $f: \mathcal{L}^{*} \rightarrow \mathcal{P}(E)$ a convex embedding, for some finite set $E$.

Then for any border $\left(\mathcal{B}^{+}, \mathcal{B}^{-}\right)$of $\left(\mathcal{L}^{*}, \preceq\right),\left(\operatorname{Max}\left(B_{0}^{+} \cup f\left(\mathcal{B}^{+}\right)\right), \operatorname{Min}\left(B_{0}^{-} \cup f\left(\mathcal{B}^{-}\right)\right)\right)$ is a polynomial extension of $\left(\mathcal{B}^{+}, \mathcal{B}^{-}\right)$in $(\mathcal{P}(E), \subseteq)$.

Proof. It suffices to notice that $\left(\mathcal{B}^{+}, \mathcal{B}^{-}\right)$is a border of $\left(\mathcal{L}^{*}, \preceq\right)$ iff $\left(f\left(\mathcal{B}^{+}\right), f\left(\mathcal{B}^{-}\right)\right)$ is a border of $\left(\mathcal{P}(E) \backslash\left(\downarrow B_{0}^{+} \cup \uparrow B_{0}^{-}\right), \subseteq\right)$ since $\left(\mathcal{L}^{*}, \preceq\right)$ is isomorphic to $(\mathcal{P}(E) \backslash(\downarrow$ $\left.\left.B_{0}^{+} \cup \uparrow B_{0}^{-}\right), \subseteq\right)$.

\subsection{Polynomial reflection of posets}

We now consider posets that are not in $\mathcal{X} \mathcal{R} \mathcal{A S}$. Our idea is to transform the initial poset to a new poset over the same ground set, in order to get a convex embedding. As a consequence, two natural question arise:

(1) For a given poset $\left(\mathcal{L}^{*}, \preceq\right)$, does there exist a "polynomial reflection" $\left(\mathcal{L}^{*}, \preceq^{\prime}\right)$ such that $\left(\mathcal{L}^{*}, \preceq^{\prime}\right)$ belongs to $\mathcal{E} \mathcal{X} \mathcal{R} \mathcal{A}$ ?

(2) How to quantify the "lost comparabilities" induced by a reflection?

In the sequel, we study poset reflection to give answers to the previous questions. Since the reflection of a poset induces the lost of some comparabilities in the original poset, we have to recover them efficiently.

Before that, we consider different examples of posets over sequences. 
Examples with different posets of sequences Let us consider sequences with or without wildcard (denoted $\star$ ), see e.g. [15].

Let $\Sigma$ be an alphabet. A sequence is an element of $\Sigma^{*}$ and a rigid sequence an element of $(\Sigma \cup\{\star\})^{*}$ of the form $P=P[1] \cdots P[m]$ such that $P[1] \neq \star$ and $P[m] \neq \star$. Let $\Sigma_{R}^{*}$ be the set of rigid sequences and $\Sigma^{*}$ the set of sequences. We denote by $\Sigma^{n}$ the set of all sequences in $\Sigma^{*}$ of size at most $n$.

Different partial orders over $\Sigma_{R}^{*}$ and $\Sigma^{*}$ exist. Let us first consider sub-word (resp. factor and prefix) posets over $\Sigma^{*}$, denoted $\left(\Sigma^{*}, \leq_{s}\right)\left(\operatorname{resp} .\left(\Sigma^{*}, \leq_{f}\right)\right.$ and $\left.\left(\Sigma^{*}, \leq_{p}\right)\right)$. Let $P[1 . . m], Q[1 . . n] \in \Sigma^{*}$. We have:

$-P \leq_{s} Q$ if there exists integers $i_{1}<\ldots<i_{m}$ in $[1 . . n]$ such that $P[j]=Q\left[i_{j}\right]$ for all $j \in[1 . . m]$.

$-P \leq_{f} Q$ if $P \leq_{s} Q$ and $i_{j}=i_{j+1}-1$ for all $j \in[1 . . m-1]$

- $P \leq_{p} Q$ if $P \leq_{f} Q$ and $i_{m}=m$.

These different posets are illustrated in Figure 4 on a simple example.

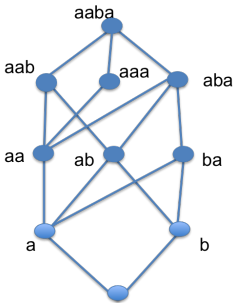

(a)

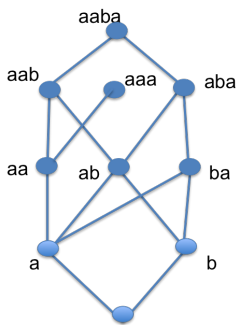

(b)

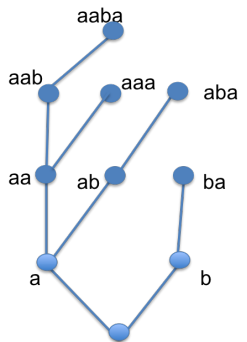

(c)

Fig. 4. (a) The sub word poset induced by the word w=aaba on $\Sigma=\{a, b\}$; (b) the factor poset which is a reflection of (a); and (c) the prefix poset which is a reflection of posets (b) and (a).

In Figure 4, let us consider the set $\{a a, a b\}$ for the different posets. Its dual set is equal to $\{b a, a a b, a a a\}$ for $(a)$ and $(b)$ and $\{b, a a b, a a a, a b a\}$ for $(c)$.

Second, we consider two posets for rigid sequences, one similar to the factor poset and another one to the prefix poset, denoted $\left(\Sigma_{R}^{*}, \sqsubseteq\right)$ and $\left(\Sigma_{R}^{*}, \sqsubseteq_{1}\right)$. Let $P[1 . . m], Q[1 . . n] \in \Sigma_{R}^{*}$. We have:

$-P \sqsubseteq Q$ if there exists $p \in[1 . . n]$ such that for every $i \in[1 . . m]$, either $P[i]=$ $Q[p+i-1]$ or $P[i]=\star$

- $P \sqsubseteq{ }_{1} Q$ if for every $i \in[1 . . m]$, either $P[i]=Q[i]$ or $P[i]=\star$

These two posets are illustrated in Figure 5, where the poset $(b)$ suggests the existence of hidden hypercubes in the poset $(a)$.

Now, we claim that the posets $\left(\Sigma^{*}, \leq_{s}\right),\left(\Sigma^{*}, \leq_{f}\right),\left(\Sigma^{*}, \leq_{p}\right)$ and $\left(\Sigma_{R}^{*}, \sqsubseteq\right)$ do not have any convex embedding. Consider again the example given in Figure 4 and the two following sets: $A=\{a, b, a b, b a\}$ and $A^{\prime}=\{a, a a, a a a\}$. 


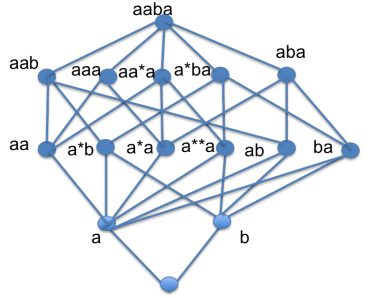

(a)

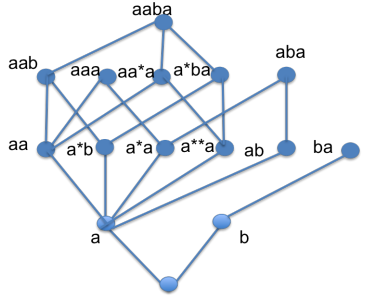

(b)

Fig. 5. (a) The factor poset of rigid sequences induced by the word w=aaba on $\Sigma=$ $\{a, b\}$; (b) the prefix poset of rigid sequences which is a reflection of posets (a).

- $A$ is convex in $\left(\Sigma^{*}, \leq_{s}\right.$ ) (Figure 4.(a)) but its image by any embedding cannot be convex in $(\mathcal{P}(E), \subseteq)$ since $(\mathcal{P}(E), \subseteq)$ is a lattice. The same reasoning applies for $\left(\Sigma^{*}, \leq_{f}\right)$ and $\left(\Sigma_{R}^{*}, \sqsubseteq\right)$.

- $A^{\prime}$ is convex in $\left(\Sigma^{*}, \leq_{p}\right)$ but its image by any embedding cannot be convex in $(\mathcal{P}(E), \subseteq)$ since $\mathcal{P}(E)$ cannot contain a convex set which is a chain of length 3 .

However, Figure 5 also shows a reflection that leads to a convex embedding. Consider $\left(\Sigma_{R}^{n}, \sqsubseteq_{1}\right)$. Let $f: \Sigma_{R}^{n} \backslash\{\epsilon\} \rightarrow \mathcal{P}(E)$ be an embedding, for some finite set $E[15,8]: f$ associates to each letter of a sequence a couple (indice, letter). For instance, let $a b$ and $b a$ be two patterns. Then $f(a b)=\{(1, a),(2, b)\}$ and $f(b a)=\{(1, b),(2, a)\}$. It is easy to verify that $f\left(\Sigma_{R}^{n} \backslash\{\epsilon\}\right)$ is convex in $(\mathcal{P}(E), \subseteq)$ [8]. Let us again consider $A=\{a, b, a b, b a\}$ : we only have $a \sqsubseteq_{1} a b, b \sqsubseteq_{1} b a$, i.e. two comparabilities ( $\left.a \Xi_{1} b a, b \Xi_{1} a b\right)$ are lost, allowing to reach the convexity constraint.

These examples point out that we have to study poset reflection to be able to obtain some convex embedding.

\subsection{Reaching convexity by poset reflection}

As shown in previous examples involving sequences, whenever possible, we have now to quantify the lost comparabilities induced by a poset reflection.

For a given element of a poset, we define its successors and its predecessors induced by a poset reflection.

Definition 4. Let $\left(P, \leq^{\prime}\right)$ a reflection of a poset $(P, \leq)$ and $x \in P$. The lost predecessors of $x$ in the reflection of $(P, \leq)$ to $\left(P, \leq^{\prime}\right)$, denoted by LostPred $(x)$, are defined by:

LostPred $(x)=\operatorname{Max}_{\leq^{\prime}}\left\{y \in P \mid y \leq x, y \nless^{\prime} x\right\}$. Similarly, the lost successors are defined by: LostSucc $(x)=\operatorname{Min}_{\leq}\left\{y \in P \mid x \leq y, x \nless^{\prime} y\right\}$.

By extension, we note LostPred $(X)=\bigcup_{x \in X} \operatorname{LostPred}(x)($ resp. LostSucc $(X))$ for $X \subseteq P$. 
Example 1. Let us consider the reflection $\left(\Sigma_{R}^{n}, \sqsubseteq_{1}\right)$ of $\left(\Sigma_{R}^{n}, \sqsubseteq\right)$ [8]. Let $S \in \Sigma_{R}^{n}$. We have LostPred $(S)=\{S[i .|S|]|1 \leq i \leq| S \mid, S[i] \neq \star\}$ and $\operatorname{LostSucc}(S)=$ $\{x \underbrace{\star \ldots \star}_{i} S|0 \leq i \leq n-| S \mid-1, x \in \Sigma\}$.

For instance with $n=5, \operatorname{LostPred}(a \star b a)=\{a \star b a, b a, a\}$ and LostSucc $(b a)=$ $\{a b a, b b a, a \star b a, b \star b a, a \star \star b a, b \star \star b a\}$.

As shown in the following lemma, we can recover the initial poset from any reduced poset with LostPred and LostSucc.

Lemma 1. Let $x \in P$ and $\left(P, \leq^{\prime}\right)$ a reflection of $(P, \leq)$. Then:

1. $\downarrow \leq x=\downarrow \leq ' \operatorname{LostPred}(x)$ and

2. $\uparrow \leq x=\uparrow \leq{ }^{\prime} \operatorname{LostSucc}(x)$.

Proof. (1) Let $y \in \downarrow \leq x$. We have either $y \leq^{\prime} x$ or $y \nless^{\prime} x$. If $y \leq \leq^{\prime} x$, then $y \in \downarrow \leq \leq^{\prime} \operatorname{LostPred}(x)$ since $x \in \operatorname{LostPred}(x)$. If $y \nless^{\prime} x$, then there exists $z \in P$ such that $y \leq \leq^{\prime} z, z \in \operatorname{LostPred}(x)$, i.e. $y \in \downarrow^{\leq^{\prime}} \operatorname{LostPred}(x)$. The same reasoning applies for (2).

Some remarks have to be made: First, for any poset, there always exists a reflection that has a convex embedding into a boolean lattice. It suffices to take a reflection which is an antichain, i.e. that deletes all comparabilities. In this case, the number of lost comparabilities can be exponential in the size of the description of the poset. Second, we would like to be able to recover lost comparabilities in polynomial time. This is formalized with the notion of polyreflection as follows.

Definition 5. $\left(\mathcal{L}^{*}, \preceq^{\prime}\right)$ is a poly-reflection of $\left(\mathcal{L}^{*}, \preceq\right)$ if $\left(\mathcal{L}^{*}, \preceq^{\prime}\right)$ is a reflection of $\left(\mathcal{L}^{*}, \preceq\right)$ and for all $x \in \mathcal{L}^{*}, \operatorname{LostPred}(x)$ and LostSucc $(x)$ are computable in polynomial time in the size of the description $\mathcal{L}$.

Example 2. Continuing the previous example, for all $S \in \Sigma_{R}^{n}$, $\operatorname{LostPred}(S)$ is polynomial in $n$ and for all $s \sqsubseteq S$, there exists $s^{\prime} \in \operatorname{LostPred}(S) \operatorname{such}$ that $s \sqsubseteq_{1} s^{\prime}$. Therefore, $\left(\Sigma_{R}^{n}, \sqsubseteq_{1}\right)$ is a poly-reflection of $\left(\Sigma_{R}^{n}, \sqsubseteq\right)$.

Now we show the relationship between borders in a poset and its reflection. For a given border on the initial poset, we define its extension in the reduced poset to take into account lost comparabilities.

Definition 6. Let $\left(\mathcal{L}^{*}, \preceq^{\prime}\right)$ be a poly-reflection of $\left(\mathcal{L}^{*}, \preceq\right)$ and $\left(\mathcal{B}^{+}, \mathcal{B}^{-}\right)$a border of $\left(\mathcal{L}^{*}, \preceq\right)$. The extension of $\left(\mathcal{B}^{+}, \mathcal{B}^{-}\right)$in $\left(\mathcal{L}^{*}, \preceq^{\prime}\right)$, denoted by $\left(\operatorname{ext}\left(\mathcal{B}^{+}\right), \operatorname{ext}\left(\mathcal{B}^{-}\right)\right)$, is defined by:

$\operatorname{ext}\left(\mathcal{B}^{+}\right)=\operatorname{Max}_{\leq}\left\{\operatorname{LostPred}(x) \mid x \in \mathcal{B}^{+}\right\}$

$\operatorname{ext}\left(\mathcal{B}^{-}\right)=\operatorname{Min}_{\leq}\left\{\operatorname{Lost\operatorname {Succ}}(x) \mid x \in \mathcal{B}^{-}\right\}$.

The "preservation" of borders can now be formally stated.

Proposition 4. Let $\left(\mathcal{L}^{*}, \preceq^{\prime}\right)$ be a poly-reflection of $\left(\mathcal{L}^{*}, \preceq\right)$ and $\left(\mathcal{B}^{+}, \mathcal{B}^{-}\right)$a border of $\left(\mathcal{L}^{*}, \preceq\right)$. Then $\left(\operatorname{ext}\left(\mathcal{B}^{+}\right), \operatorname{ext}\left(\mathcal{B}^{-}\right)\right)$is a polynomial extension of $\left(\mathcal{B}^{+}, \mathcal{B}^{-}\right)$. 
Proof. We have to show:

1. $\left(\operatorname{ext}\left(\mathcal{B}^{+}\right), \operatorname{ext}\left(\mathcal{B}^{-}\right)\right)$is a border of $\left(\mathcal{L}^{*}, \preceq^{\prime}\right)$ with $\mathcal{B}^{+} \subseteq \operatorname{ext}\left(\mathcal{B}^{+}\right)$and $\mathcal{B}^{-} \subseteq$ $\operatorname{ext}\left(\mathcal{B}^{-}\right)$

2. $\left|\operatorname{ext}\left(\mathcal{B}^{+}\right)\right|+\left|\operatorname{ext}\left(\mathcal{B}^{-}\right)\right|$is polynomial in $\left|\mathcal{B}^{+}\right|+\left|\mathcal{B}^{-}\right|$.

(1) Any reflection preserves all incomparabilities and $x \in \operatorname{LostPred}(x)$ (resp $x \in \operatorname{LostSucc}(x))$ for all $x \in \mathcal{L}^{*}$. Then $\mathcal{B}^{+} \subseteq \operatorname{ext}\left(\mathcal{B}^{+}\right)$and $\mathcal{B}^{-} \subseteq \operatorname{ext}\left(\mathcal{B}^{-}\right)$. By definition, $\operatorname{ext}\left(\mathcal{B}^{+}\right)$and $\operatorname{ext}\left(\mathcal{B}^{-}\right)$are antichains in $\left(\mathcal{L}^{*}, \preceq^{\prime}\right)$. By Lemma 1 , the result follows.

$(2)\left|\operatorname{ext}\left(\mathcal{B}^{+}\right)\right|+\left|\operatorname{ext}\left(\mathcal{B}^{-}\right)\right|$is polynomial in $\left|\mathcal{B}^{+}\right|+\left|\mathcal{B}^{-}\right|$since $\left(\mathcal{L}^{*}, \preceq^{\prime}\right)$ is a polyreflection of $\left(\mathcal{L}^{*}, \preceq\right)$ since computing LostPred $(x)$ and LostSucc $(x)$ can be done in polynomial time in the size of the description of $\mathcal{L}$.

The notion of poly-reflection allows to define the last class of posets, called $\mathcal{E} \mathcal{W} \mathcal{R} \mathcal{A S}$, meaning Efficient weak representation as sets. $\mathcal{E} \mathcal{W} \mathcal{A} \mathcal{S}$ is the more general class ensuring the existence of quasi-polynomial algorithms. It combines both poly-reflection of posets and $\mathcal{E} \mathcal{X} \mathcal{R} \mathcal{A}$.

Definition 7. $\left(\mathcal{L}^{*}, \preceq\right) \in \mathcal{E} \mathcal{W} \mathcal{R} \mathcal{A S}$ iff there exists a poly-reflection $\left(\mathcal{L}^{*}, \preceq^{\prime}\right)$ of $\left(\mathcal{L}^{*}, \preceq\right)$ such that $\left(\mathcal{L}^{*}, \preceq^{\prime}\right) \in \mathcal{E} \mathcal{X} \mathcal{R} \mathcal{A S}$.

Then, this definition means that if some comparabilities can be forgotten up to a polynomial cost to recover them - to get a new poset satisfying the condition of $\mathcal{E} \mathcal{X} \mathcal{R} \mathcal{S}$, then the dualization problem on the initial poset can be reduced to DualizationOnSet.

Example 3. Continuing previous examples, we have $\left(\Sigma_{R}^{*}, \sqsubseteq_{1}\right)$ is a poly-reflection of $\left(\Sigma_{R}^{*}, \sqsubseteq\right)$ and $\left(\Sigma_{R}^{*}, \sqsubseteq_{1}\right)$ belongs to $\mathcal{E} \mathcal{X} \mathcal{R} \mathcal{A S}$. Then, $\left(\Sigma_{R}^{*}, \sqsubseteq\right)$ belongs to $\mathcal{E} \mathcal{W} \mathcal{R} \mathcal{A S}$.

The main result concerning the $\mathcal{E} \mathcal{W R} \mathcal{A S}$ class is now given:

Theorem 1. Let $\left(\mathcal{L}^{*}, \preceq\right) \in \mathcal{E} \mathcal{W} \mathcal{R} \mathcal{A S}$. Assume that $\left(\mathcal{L}^{*}, \preceq^{\prime}\right)$ is a poly-reflection of $\left(\mathcal{L}^{*}, \preceq\right)$ such that $\left(\mathcal{L}^{*}, \preceq^{\prime}\right)$ belongs to $\mathcal{E} \mathcal{X} \mathcal{R} \mathcal{A S}$, i.e. $\left(\mathcal{L}^{*}, \preceq^{\prime}\right)$ isomorphic to $\mathcal{P}(E) \backslash\left(B_{0}^{+} \cup B_{0}^{-}\right)$. Then, for any border $\left(\mathcal{B}^{+}, \mathcal{B}^{-}\right)$of $\left(\mathcal{L}^{*}, \preceq\right)$ :

- $\left(B_{0}^{+} \cup f\left(\operatorname{ext}\left(\mathcal{B}^{+}\right)\right), B_{0}^{-} \cup f\left(\operatorname{ext}\left(\mathcal{B}^{-}\right)\right)\right)$is a border in $(\mathcal{P}(E), \subseteq)$,

$-\left(B_{0}^{+} \cup f\left(\operatorname{ext}\left(\mathcal{B}^{+}\right)\right), B_{0}^{-} \cup f\left(\operatorname{ext}\left(\mathcal{B}^{-}\right)\right)\right)$is a polynomial extension of $\left(\mathcal{B}^{+}, \mathcal{B}^{-}\right)$,

The following corollary gives the relationship between all the classes introduced so far.

Corollary 1. Let $\left(\mathcal{L}^{*}, \preceq^{\prime}\right)$ be a poly-reflection of $\left(\mathcal{L}^{*}, \preceq\right)$ such that $\left(\mathcal{L}^{*}, \preceq^{\prime}\right)$ belongs to $\mathcal{X} \mathcal{R} \mathcal{A S}$, i.e. $\left(\mathcal{L}^{*}, \preceq^{\prime}\right)$ isomorphic to $\mathcal{P}(E) \backslash\left(B_{0}^{+} \cup B_{0}^{-}\right)$. We have:

1. $\left(\mathcal{L}^{*}, \preceq\right) \in \mathcal{R} \mathcal{A S}$ if $\left(\mathcal{L}^{*}, \preceq\right)=\left(\mathcal{L}^{*}, \preceq^{\prime}\right)$ and $B_{0}^{+}=B_{0}^{-}=\emptyset$.

2. $\left(\mathcal{L}^{*}, \preceq\right) \in \mathcal{X} \mathcal{R} \mathcal{A S}$ if $\left(\mathcal{L}^{*}, \preceq\right)=\left(\mathcal{L}^{*}, \preceq^{\prime}\right)$.

3. $\left(\mathcal{L}^{*}, \preceq\right) \in \mathcal{E} \mathcal{X} \mathcal{R} \mathcal{A S}$ if $\left(\mathcal{L}^{*}, \preceq\right)=\left(\mathcal{L}^{*}, \preceq^{\prime}\right)$ and the size of $B_{0}^{+} \cup B_{0}^{-}$is polynomial in the size of the description of $\left(\mathcal{L}^{*}, \preceq\right)$.

4. $\left(\mathcal{L}^{*}, \preceq\right) \in \mathcal{E} \mathcal{W R} \mathcal{A S}$ if the size of $B_{0}^{+} \cup B_{0}^{-}$is polynomial in the size of the description of $\left(\mathcal{L}^{*}, \preceq\right)$. 


\subsection{DualizeOnSeq is equivalent to DualizeOnSet}

Recall that we consider rigid sequences only. The dualization problem can be stated as follows:

\section{DualizeOnSeq}

Input: $\Sigma$ a totally ordered alphabet with a minimal element $\star, n$ a positive integer, $B^{+}$a positive border of $\Sigma_{R}^{n}$.

output: $B^{-}$such that $\left(B^{+}, B^{-}\right)$is a border of $\Sigma_{R}^{n}$.

We have already showed that DualizeOnSeq is at least as hard as DualizeOnSet in [8]. In the sequel, we point out that DualizeOnSet is at least as hard as DualizeOnSeq, and therefore the two problems are polynomially equivalent. Indeed, we show that DualizeOnSet is a particular case of DualizeOnSeq.

Let $\Sigma=\{1,2, \ldots, n, \star\}$ be an ordered alphabet (i.e. $\star<1<2 \ldots<n$ and $S \in \Sigma^{n}$. The sequence $S$ is said to be an ordered sequence if for any $i, j \in[1 . . n]$ such that $i<j, S[i] \neq \star$ and $S[j] \neq \star$ we have $S[j]-S[i]=j-i$. We denote $\Sigma_{O}^{n} \subseteq \Sigma^{n}$ the set of all ordered sequences of size at most $n$. For example, the sequence $2 \star \star 5$ is an ordered sequence but $2 \star 5$ is not.

The following lemma characterizes ordered sequences.

Lemma 2. Let $\Sigma=\{1,2, \ldots, n, \star\}$ be an ordered alphabet and $S \in \Sigma^{n}$. Then $S \in \Sigma_{O}^{n}$ iff $S$ does not contain a subsequence of the form $i \underbrace{\star \ldots \star}_{k} j$ with either $i<j$ and $k \neq j-i-1$, or $i \geq j$ with $k \in[0 . . n-2]$.

Consider the set $B_{0}^{-}=\left\{i *^{k} j \quad \mid i \geq j, k \in[0 . . n-2]\right\} \cup\left\{i *^{k} j \mid i<\right.$ $j, k \in[0 . . n-2], k \neq j-i-1\}$. For example for $\Sigma=\{1,2,3, *\}$ we have $B_{0}^{-}=$ $\{11,1 * 1,22,2 * 2,33,3 * 3,21,2 * 1,31,3 * 1,32,3 * 2\} \cup\{13\}$.

Lemma 3. $\Sigma^{n} \backslash \uparrow B_{0}^{-}=\Sigma_{O}^{n}$.

Let $V=\{1, \ldots, n\}$ be a set. We define the mapping $f: \mathcal{P}(V) \rightarrow \Sigma^{n}$ such that for any $E \in \mathcal{P}(V), f(E)=S$ with $S[i]=i$ if $i \in E$ and $S[i]=\star$ otherwise. Without loss of generality, we delete the symbols $\star$ that are prefix or suffix of $f(E)$. Note that $f(E)[i]=\star$ means that $i \notin E$. For example $f(\{2,5\})=2 \star \star 5$ and $f(\{\})$ is the empty sequence.

Proposition 5. Let $V=\{1, \ldots, n\}$ be a set. Then the mapping $f$ is a convex embedding of $\mathcal{P}(V)$ into $\Sigma^{n}$. Moreover $f(\mathcal{P}(V))=\Sigma_{O}^{n}$.

Proof. Let $P, Q$ be two sequences that are images of two sets $A \subset B \subseteq V$, i.e. $f(A)=P$ and $f(B)=Q$. Clearly $f(A) \sqsubset f(B)$.

Now suppose there is a sequence $S$ such that $P \sqsubset S \sqsubset Q$.

For every $i \neq j \in[1 . . n]$ we have either $S[i] \neq S[j]$ or $S[i]=S[j]=\star$, by definition of the embedding $f$. Then the set $C=\{x \in V \mid S[i]=x, i \in[1 . . n]\}$ is clearly defined. Moreover $f(C)=S$ and $A \subset C \subset B$, since for any $x \in \Sigma$, $x \not \subset \star$, but $\star \sqsubset x$.

We have $f(\mathcal{P}(V))=\Sigma_{O}^{n}$ by construction. 
Theorem 2. DualizeOnSeq and DualizeOnSet are polynomially equivalent.

Proof. We have shown that DualizeOnSeq is polynomially reducible to DualizeOnSet [8]. In Proposition 5, we have shown the existence of a convex embedding from $\mathcal{P}(V)$ into $\Sigma^{n}$. Moreover we have shown that $B_{0}^{+}=\emptyset$ and according to Lemma 2 the size of $B_{0}^{-}$is bounded by $O\left(n^{3}\right)$. Thus DualizeOnSet is polynomially reducible to DualizeOnSeq.

We have shown that the dualization on rigid sequences with wildcard is equivalent to the dualization on set, i.e. enumerating minimal transversals of a given hypergraph.

\section{References}

1. Eiter, T., Gottlob, G.: Identifying the minimal transversals of a hypergraph and related problems. SIAM J. Comput. 24(6) (1995) 1278-1304

2. Fredman, M.L., Khachiyan, L.: On the complexity of dualization of monotone disjunctive normal forms. J. Algorithms 21(3) (1996) 618-628

3. Eiter, T., Gottlob, G., Makino, K.: New results on monotone dualization and generating hypergraph transversals. SIAM J. Comput. 32 (February 2003) 514537

4. Elbassioni, K.M.: Algorithms for dualization over products of partially ordered sets. SIAM J. Discrete Math. 23(1) (2009) 487-510

5. Kanté, M.M., Limouzy, V., Mary, A., Nourine, L.: On the enumeration of minimal dominating sets and related notions. Revised version submitted (2013)

6. Mannila, H., Toivonen, H.: Levelwise search and borders of theories in knowledge discovery. Data Mining and Knowledge Discovery 1(3) (1997) 241-258

7. Gunopulos, D., Khardon, R., Mannila, H., Saluja, S., Toivonen, H., Sharm, R.S.: Discovering all most specific sentences. ACM Trans. Database Syst. 28(2) (2003) 140-174

8. Nourine, L., Petit, J.M.: Extending Set-Based Dualization: Application to Pattern Mining. In Press, I., ed.: ECAI 2012. (August 2012)

9. Elbassioni, K.: Incremental algorithms for enumerating extremal solutions of monotone systems of submodular inequality and their applications. Phd thesis, Rutgers, The state university of New jersey (2002)

10. Davey, B.A., Priestley, H.A.: Introduction to Lattices and Order. Cambridge Press (1990)

11. Ganter, B., Wille, R.: Formal Concept Analysis. Springer-Verlag (1999)

12. Agrawal, R., Imielinski, T., Swami, A.: Mining associations between sets of items in massive databases. In: ACM SIGMOD 1993, Washington D.C. (1993) 207-216

13. Mannila, H., Rih, K.J.: Algorithms for inferring functional dependencies from relations. Data and Knowledge Engineering 12(1) (February 1994) 83-99

14. De Marchi, F., Petit, J.M.: Zigzag: a new algorithm for mining large inclusion dependencies in databases. In: ICDM'03, USA. (November 2003) 27-34

15. Arimura, H., Uno, T.: Polynomial-delay and polynomial-space algorithms for mining closed sequences, graphs, and pictures in accessible set systems. In: SDM. (2009) 1087-1098 